\title{
System-wide learning from root cause analysis: a report from the New South Wales Root Cause Analysis Review Committee
}

\author{
Jonny Taitz, ${ }^{1}$ Kelvin Genn, ${ }^{2}$ Vanessa Brooks, ${ }^{2}$ Deborah Ross, ${ }^{2}$ Kathleen Ryan, ${ }^{2}$ \\ Bronwyn Shumack, ${ }^{1}$ Tony Burrell, ${ }^{1}$ Peter Kennedy, ${ }^{1}$ on behalf of the NSW RCA Review \\ Committee
}

${ }^{1}$ Clinical Excellence Commission, Martin Place, Sydney, New South Wales, Australia ${ }^{2}$ Quality and Safety Branch, NSW Department of Health, North Sydney, New South Wales, Australia

\section{Correspondence to} Dr Jonny Taitz, Level 3 Executive Unit, Sydney Children's Hospital, High Street, Randwick, Sydney 2031, Australia; jonny.taitz@sesiahs. health.nsw.gov.au

Accepted 2 November 2009 Published Online First 29 July 2010

\section{ABSTRACT}

Background Preventable errors are common in healthcare. Over the last decade, Root Cause Analysis (RCA) has become a key tool for healthcare services to investigate adverse events and try to prevent them from happening again. The purpose of this paper is to highlight the work of the New South Wales (NSW) RCA Review Committee. The benefits of correctly classifying, aggregating and disseminating RCA data to clinicians will be discussed. In NSW, we perform an average of 500 RCAs per year. It is estimated that each RCA takes between 20 and $90 \mathrm{~h}$ to perform. In 2007, the NSW Clinical Excellence Commission (CEC) and the Quality and Safety Branch at the Department of Health constituted an RCA review committee. 445 RCAs were reviewed by the committee in 14 months. 41 RCAs were related to errors in managing acute coronary syndrome.

Results and discussion The large number of RCAs has enabled the committee to identify emerging themes and to aggregate the information about underlying human (staff), patient and system factors. The committee has developed a taxonomy based on previous work done within health and aviation and assesses each RCA against this set of criteria. The effectiveness of recommendations made by RCA teams requires further review. There has been conjecture that staff do not feel empowered to articulate root causes which are beyond the capacity of the local service to address.

Conclusion Given the number of hours per RCA, it seems a shame that the final output of the process may not in fact achieve the desired patient safety improvements.

\section{INTRODUCTION}

The Institute of Medicine's landmark publication To Err is Human: Building a Safer Health System ${ }^{1}$ highlighted concern about patient injuries received during healthcare both in the USA and internationally. Prior to this publication, quality improvement in the health industry was regarded as peripheral to the main task of treating patients, despite the ever increasing complexity of healthcare. The IOM report changed the way healthcare managers and professionals regard medical errors and injury. ${ }^{1-3}$ Preventable errors are still common in healthcare. Those that lead to injury are commonly referred to as adverse events. The purpose of this paper is to highlight the work of the NSW RCA review committee. In particular, the benefits of correctly classifying, aggregating and disseminating Root Cause Analysis (RCA) data to clinicians will be discussed.

Over the last decade, RCA has become a key tool for healthcare services to better understand adverse events. ${ }^{4}$ It is a structured methodology that was originally used in high-risk industries such as nuclear power and aviation. ${ }^{5-8}$ Although RCA is now widely used as an error-analysis tool in healthcare, discussion of RCA did not emerge in the patient safety and medical literature until the mid to late 1990s. The underpinning principle of RCA is that the propensity of humans to make errors cannot be eliminated, and instead of blaming individuals we need to identify and rectify the underlying system vulnerabilities which allow human error to cause harm to patients. The original pioneers were Bagian ${ }^{9}$ at the US department of Veteran Affairs (VA) and Croteau at the Joint Commission. Although each organisation developed a slightly different approach, it was the first time that an attempt at systematic review of medical error was made. ${ }^{8}$ Other pioneering work was undertaken by Vincent, although his work revolved less around root causes for an event and more on the investigation itself. ${ }^{10}$ Many countries have adopted the RCA methodology to improve patient safety. Early adopters have included New South Wales and Queensland in Australia, Denmark and The National Patient Safety Agency in England. 81112

In 1999, Bagian' enhanced the RCA process through the introduction of specific nomenclature in the VA patient safety programme. The RCA methodology was adjusted to answer three questions that could make organisations error wise: What happened? Why did it happen? What can be done to prevent it from happening again ${ }^{8912-15}$ Currently, the Joint Commission requires healthcare organisations in the USA to perform an RCA for every sentinel event, while in the VA system, facilities are required to submit a report to the National Centre for Patient Safety (NCPS) for serious actual (and potential) events. ${ }^{4}$ To date, the VA has conducted over 10000 RCAs, and The Joint Commission has mandated that over 4000 RCAs be performed.

In NSW, RCAs are required by legislation for every SAC1clinical incident, as classified by the Severity Assessment Code (SAC) chart, and we perform an average of 500 clinical management RCAs per year. All clinical SAC1 RCA reports are required to be submitted to NSW Health. In comparison, the 
health services in Denmark has conducted 235 RCAs in a 6-year period from 2002 to $2008 .^{16}$

While there are many reported/incidental benefits of conducting an RCA, there is very little scientific evidence on whether these thousands of RCAs have improved the quality of clinical care. The translation of RCA findings into effective training and educational strategies also remains unclear. ${ }^{17-19}$ This paper aims to show that lessons can be learnt through aggregating and disseminating RCA data. We believe that this information can help clinicians improve their practice and also help the system address major state wide patient safety issues.

\section{NSW RCA COMMITTEE}

In 2007, in response to concerns that there was very little evidence of system-wide learning following incident investigations, the NSW Clinical Excellence Commission (CEC) and the Quality and Safety Branch at the Department of Health constituted an RCA Review Committee to look at issues related to clinical management and some sentinel events. Maternal/ perinatal care and suspected suicide of mental health patients were not included. The committee is a now a key component of the Patient Safety and Clinical Quality Program in NSW. ${ }^{20}$ It comprises a multidisciplinary group of clinicians, patient safety managers, directors of clinical governance as well as staff from the Quality and Safety Branch (NSW Health) and the CEC.

Initially, the review of RCAs was based on the existing categories within the NSW Incident and Information Management System (IIMS). Some classification was also contained within the RCA reports, but was limited to seven specific categories, ranging from 'communication' to 'safety mechanisms.'

The committee reviewed an average of 15 RCAs per session. It soon became apparent that further analysis of the information contained within the reports was necessary to better quantify identified risks into subsets of patient, human and system factors. It would also assist in monitoring the number of incidents related to particular clinical conditions.

\section{REVIEW OF WORK DONE BY THE COMMITTEE}

The findings of the committee are highlighted in figure 1 and tables $1-4$.
The review of data also prompted specific analyses to further explore emerging themes in the RCAs. We used the state-wide IIMS data to conduct searches of the existing fields as well as free text analysis. The CEC has subsequently begun producing 'clinical focus reports' which explore in detail the issues identified in IIMS and RCA data for types of incidents reviewed by the RCA Review Committee. Distributing these to key clinical groups, the Department of Health and back to clinicians via the area health service Clinical Governance Units has been a very positive outcome for the Committee. These have prompted discussion at both state and local level, and have invigorated clinical groups to improve the management of conditions highlighted in the focus reports.

As expected, in any system where sentinel events include patient identification, $97(22 \%)$ of the RCAs related to procedures involving the wrong patient, wrong site or wrong surgical device. A large number were for incorrect imaging, but 10 were for wrong lens eye surgery performed across NSW. Given the high numbers of this procedure across the state, each was considered locally to be a rare event. Following feedback from the RCA committee, NSW Health consulted with the College of Ophthalmology and strengthened the policy relating to patent identification prior to lens procedures. It also became evident that staff routinely worked around inadequate policies, environmental conditions and gaps in orientation and supervision when trying to provide the right treatment for the right patient. Human error, including 'violation,' was a strong factor in this group of incidents.

Sixty-seven RCAs identified deficiencies in monitoring of patients, including basic vital sign monitoring not being performed with the regularity and completeness which would be expected. This often resulted in a failure to recognise deterioration in a patient, even though they were being monitored. The concern over failure to recognise the deteriorating patient was also highlighted in the recent Garling Enquiry into acute care services in NSW. ${ }^{21}$ Quantifying and describing this issue resulted in resources being committed to a CEC programme on the deteriorating patient--The 'Between the Flags' project.

We also recognised that recommendations were often weak and that there was no formalised system for ensuring that
Figure 1 Classification of Root Cause Analyses by percentage.

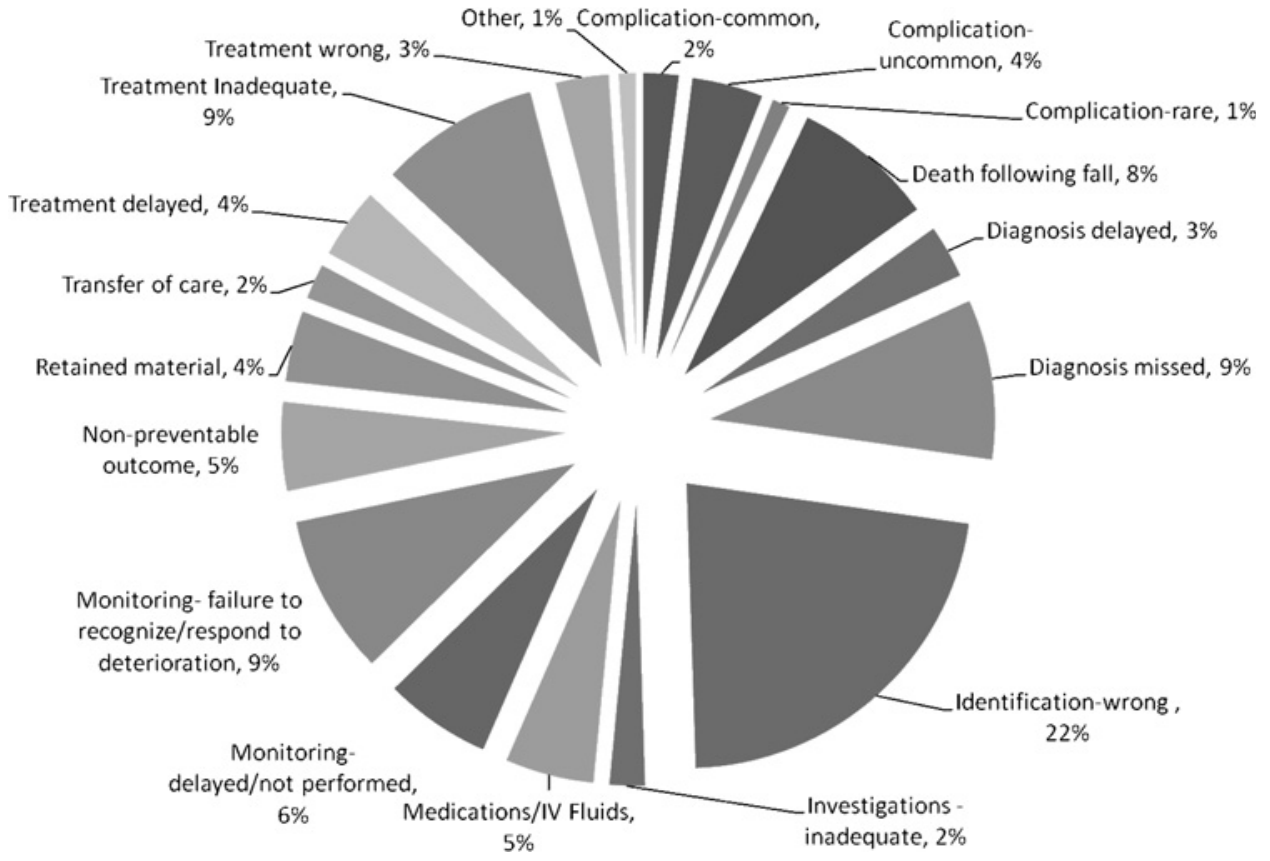

Complication-

Complication-rare, $1 \%$ 
Table 1 Clinical management incident classification set (from Incident and Information Management System)

\begin{tabular}{|c|c|c|c|c|c|}
\hline $\begin{array}{l}\text { Incident } \\
\text { classification }\end{array}$ & Descriptor & & & & \\
\hline Complication & Common & Uncommon & Rare & & \\
\hline Diagnosis & Delayed & Missed & & & \\
\hline Identification & Wrong patient & Wrong site & Wrong procedure & Wrong device/implant & \\
\hline Investigations & Delayed & Inappropriate & Results not actioned & & \\
\hline $\begin{array}{l}\text { Interhospital } \\
\text { transfer/retrieval }\end{array}$ & $\begin{array}{l}\text { Inadequate } \\
\text { stabilisation }\end{array}$ & Delayed & & & \\
\hline $\begin{array}{l}\text { Monitoring/ } \\
\text { observations }\end{array}$ & Not performed & Not reviewed & $\begin{array}{l}\text { Significance not } \\
\text { recognised }\end{array}$ & $\begin{array}{l}\text { Delay/failure to } \\
\text { recognise deterioration }\end{array}$ & $\begin{array}{l}\text { Inappropriate response } \\
\text { to escalation }\end{array}$ \\
\hline Retained material & Retained instrument & Incorrect count & & & \\
\hline Transfer of care & Inadequate handover & Inadequate planning & Timeliness/appropriateness & & \\
\hline Treatment & Delayed & Inadequate & & & \\
\hline $\begin{array}{l}\text { Other categories } \\
\text { allocated }\end{array}$ & Blood/blood products & Death following fall & $\begin{array}{l}\text { Medications/intravenous } \\
\text { fluids }\end{array}$ & Non-preventable outcome & \\
\hline
\end{tabular}

Table 2 Root Cause Analysis review committee classification subset for system, human and patient factors

\begin{tabular}{|c|c|c|}
\hline System factor & \multicolumn{2}{|l|}{ Descriptors } \\
\hline Access & \multicolumn{2}{|c|}{$\begin{array}{l}\text { Delayed or no access to service/bed/diagnostics } \\
\text { Patient located outside clinical specialty area (outlier) }\end{array}$} \\
\hline Care planning & \multicolumn{2}{|c|}{$\begin{array}{l}\text { Patient perceived as palliative; lead team/clinician not deter- } \\
\text { mined } \\
\text { Patient/carer not involved in care planning }\end{array}$} \\
\hline Communication & \multicolumn{2}{|c|}{$\begin{array}{l}\text { Inadequate communication between care providers; inadequate } \\
\text { information/education to patient/carer; informed consent not } \\
\text { obtained; documentation inadequate }\end{array}$} \\
\hline Equipment & \multicolumn{2}{|c|}{$\begin{array}{l}\text { Not maintained or not available (as per PIT-medical devices/ } \\
\text { equipment) }\end{array}$} \\
\hline Environment & \multicolumn{2}{|c|}{ Physical environment factors impaired care delivery } \\
\hline Policy/guidelines & \multicolumn{2}{|c|}{$\begin{array}{l}\text { Not known/not available; not implemented (including if unclear } \\
\text { or unworkable); not in line with evidence-based practise or } \\
\text { State directives }\end{array}$} \\
\hline Risk management & \multicolumn{2}{|c|}{$\begin{array}{l}\text { Known risk not mitigated (including look alike/sound alike); } \\
\text { review/monitoring systems inadequate }\end{array}$} \\
\hline $\begin{array}{l}\text { Teamwork/lead } \\
\text { clinician }\end{array}$ & \multicolumn{2}{|c|}{ Teamwork not evident; no identified lead clinician } \\
\hline Transfer & \multicolumn{2}{|c|}{ Patient unstable/unsuitable for transfer (time/service) } \\
\hline Supervision & \multicolumn{2}{|l|}{ Supervision/support inadequate } \\
\hline Workforce & \multicolumn{2}{|c|}{$\begin{array}{l}\text { Skill mix inappropriate; orientation/induction inadequate; } \\
\text { training/education inadequate; issues with credentialing/scope } \\
\text { of practice; availability of senior staff }\end{array}$} \\
\hline \multicolumn{3}{|l|}{ Human factor (staff) } \\
\hline Cognitive errors & \multicolumn{2}{|c|}{$\begin{array}{l}\text { Failure to understand/synthesise/act appropriately on available } \\
\text { information, including following wrong clinical pathway or not } \\
\text { seeking appropriate assistance; mindset/narrow thinking or } \\
\text { confined to rule-based thinking }\end{array}$} \\
\hline Violations & \multicolumn{2}{|c|}{$\begin{array}{l}\text { Risky or reckless behaviour includes: } \\
\text { - intentionally exceeding scope of practice } \\
\text { - acting outside widely accepted standards (or policy) } \\
\text { - intentionally deviating from intended use (equipment, } \\
\text { medication) }\end{array}$} \\
\hline Personal conditions & \multicolumn{2}{|c|}{$\begin{array}{l}\text { Physically fatigued or unwell; mentally fatigued, distracted or } \\
\text { unwell }\end{array}$} \\
\hline Skill-based errors & \multicolumn{2}{|c|}{$\begin{array}{l}\text { Errors of omission or commission during diagnosis, planning, } \\
\text { and treatment or general care, due to the operator not } \\
\text { completing a particular task in line with their attained skill; lapse } \\
\text { of attention or memory }\end{array}$} \\
\hline \multicolumn{3}{|c|}{ Patient factors monitored by the Committee } \\
\hline $\begin{array}{l}\text { Patient is/is } \\
\text { perceived to be 'non- } \\
\text { compliant' }\end{array}$ & $\begin{array}{l}\text { Developmentally delayed/ } \\
\text { disability }\end{array}$ & $\begin{array}{l}\text { Culturally and linguistically } \\
\text { diverse }\end{array}$ \\
\hline $75-90$ years & Emergency presentation & No advocate/support \\
\hline 90 or older & Frequent flyer & Obese \\
\hline Aboriginal & From residential care & Out of hours presentation \\
\hline Comorbidities & History of aggression & Substance misuse \\
\hline Delirium/confusion & & \\
\hline
\end{tabular}

PIT, principal incident type. proposed recommendations had been carried out in the suggested time frame. Recommendations that emphasised staff education or policy development were categorised as weak, as the committee doubted that, if implemented, the recommendations would prove effective in reducing patient harm. As a result, we developed a checklist that reviewed the strength of recommendations and whether they were implemented on time. Recommendations were categorised as weak (general education, policy development, documentation), medium (staffing changes and competency-based education) and strong (equipment changes or process redesign)-see page 3 of the RCA checklist. To date, $86 \%$ of recommendations were considered weak, $7 \%$ medium and $5 \%$ strong.

\section{IMPLICATIONS AND RECOMMENDATIONS}

The introduction of IIMS and RCA has been very effective in raising the profile of incident reporting and investigation in NSW. A key challenge remains timely feedback to clinicians at the coal face. We have used Safety Alert Broadcasts (SAB) with variable effect. The SAB is based on the Safety Notice developed by the

Table 3 Classification of Root Cause Analyses by minimum data set

\begin{tabular}{lr}
\hline & No \\
\hline Complication-common & 7 \\
Complication-uncommon & 17 \\
Complication-rare & 4 \\
Death following fall & 34 \\
Diagnosis delayed & 14 \\
Diagnosis missed & 41 \\
Identification-wrong patient/site/ & 97 \\
procedure/device & \\
Investigations-inadequate & 9 \\
Medications/intravenous fluids & 24 \\
Monitoring-delayed & 16 \\
Monitoring-failure to recognise & 30 \\
deterioration & \\
Monitoring-inappropriate response to & 12 \\
escalation & \\
Monitoring-not performed & 10 \\
Non-preventable outcome & 24 \\
Retained material & 20 \\
Transfer-inadequate & 9 \\
Treatment delayed & 19 \\
Treatment inadequate & 38 \\
Treatment wrong & 14 \\
Other & 6 \\
Total & 445 \\
\hline
\end{tabular}


Table 4 Classification of recommendations

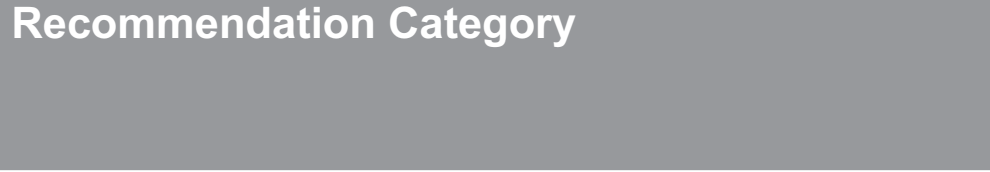

Alerts/warning/labeling

\section{Checklists}

Expected practice - no policy

Communication and documentation processes

Education - general

Education - targeted

Environmental (modifications/storage)

Equipment

Counseling/directive/memo

Organisation/management/rostering

Policies/procedures/guidelines (incl. review)

Staffing numbers or skill mix (incl. review)

Workflow or process redesign

\section{Classification}

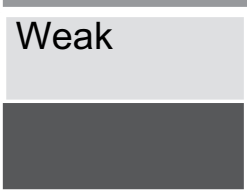

Medium

Strong
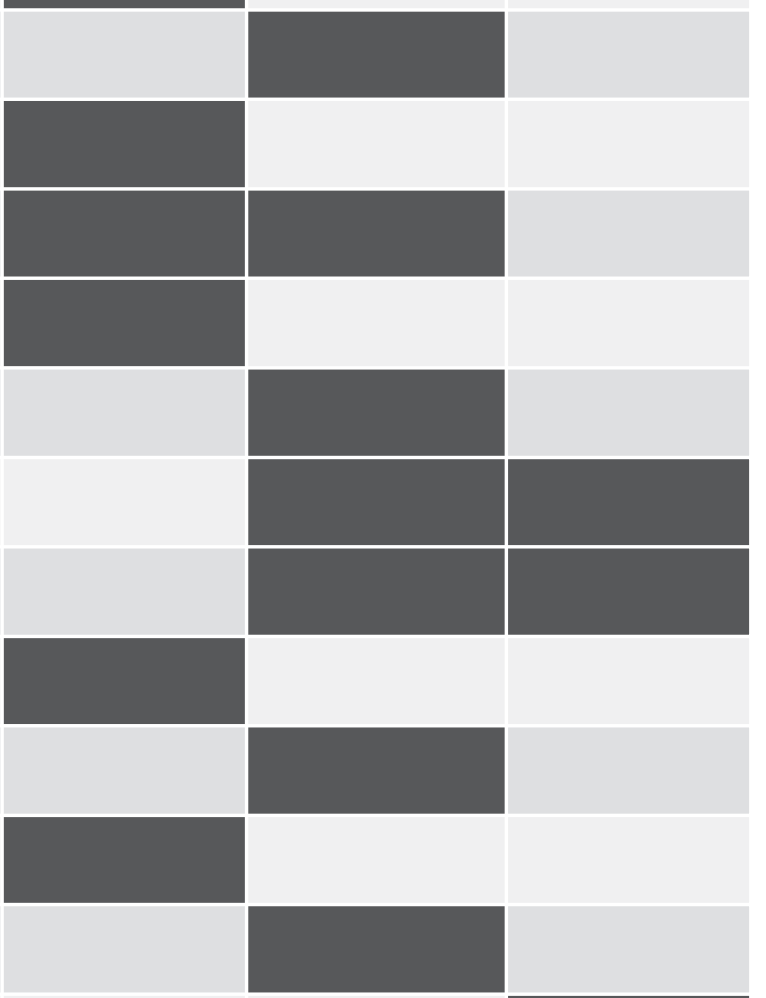

NPSA in England ${ }^{13}$ and is distributed through the Quality and Safety Branch to clinicians via the Clinical Governance units. We have, for example, used the safety broadcasts to highlight the problem of Pulmonary Embolism in young patients, misplacement of naso-gastric tubes and medication errors.

The CEC is currently trialling other feedback mechanisms including monthly bulletins and electronic modalities. The feasibility of an intranet based library of all RCAs performed in NSW and their recommendation was recommended by Garling ${ }^{21}$ and will be investigated by the CEC. We feel that a combination of communication strategies is desirable and that different adverse events allow for different dissemination strategies.

We have referred our concerns about ACS management to the Cardiac Society via the Department of Health Clinical Redesign Group. We have highlighted the importance of timely chemistry reporting and reliable ECG machines which have the capacity to provide a diagnosis, especially in smaller hospitals. To date, the cardiac society is reviewing its ACS guidelines, particularly around the need to confirm Troponin results prior to transfer of patients out of the Emergency Department.

The committee also reports monthly via its chairperson to the Directors of Clinical Governance to ensure that feedback is consistent and timely, and to open lines of communication between the committee and the Clinical Governance Units. A challenge remains to ensure that Clinical Governance Units distribute reports in a timely fashion to the clinicians. Our latest report focuses on the issue of tracheostomy tube dislodgement. The committee was able to identify seven deaths from tracheostomy tubes dislodging over a 12-month period. All occurred in tertiary settings and after hours. This equates to one every 7 weeks. We have alerted the Department of Health and also have presented potential solutions to The Intensive Care Task Group.

Our review provides further evidence of the value of ensuring routine monitoring of patients and use of a Rapid Response or Medical emergency team (MET) as a key component of a safe hospital. Added to this is a further recommendation that no patient should be transferred out of high-level care (emergency department or intensive care unit into a low acuity ward if their observations breach local MET calling criteria.

The committee remains concerned that nearly $25 \%$ of all RCAs reviewed involved incorrect patient identification. While much work has been done in the area of surgical time out and correct patient/correct procedure policies, the findings of the RCA review again highlight the need for appropriate team debriefing prior to the commencement of a procedure.

Another challenge remains reviewing implementations recommended by RCAs. To date, we have not managed to develop a robust framework to ensure that recommendations are of 
sufficient strength and that they are implemented in a timely manner. This may lead to a continued lack of error wisdom ${ }^{22}$ and frustration among clinicians and potential loss of confidence in the RCA tool. Currently, follow-up of implementation of recommendations is not a standard element of the RCA process at state level but is a responsibility of each area health service. It is noted that there are often budgetary implications which reduce the capacity of managers to endorse and implement some changes and a consequent reluctance by teams to definitively identify such issues which are beyond the control of an individual service to fix.

It is also unwise to base an entire patient safety initiative on the recommendations of a single RCA. We believe therefore that recommendations should be scrapped from the RCA methodology and in its place lessons learnt; risks and potential solutions should be identified. The risks identified should then be linked to an area's risk registry, and as these risks may relate to unit, hospital, area or state-wide, different groups may be responsible for reducing or eliminating these risks. In this way, clinicians can highlight the real issues without suggesting weak recommendations that they feel the area executive will be able to resource.

Our initial findings highlight the as yet untapped system-wide learning potential of the RCA methodology. It is clear that a single RCA in and of itself may provide little learning beyond the unit and staff involved. However, through aggregation of RCA data and successful dissemination strategies, healthcare workers can learn about adverse events rapidly.

One of the key lessons learnt from our committee is the value of a multidisciplinary governing body accepting responsibility for aggregating incident data and disseminating findings widely in that system or country. Critical to the process would be to review the risks identified, potential solutions and lessons learnt from individual RCAs and develop an evidence-based evaluation tool to gauge whether risks identified have led to improved patient safety on a system-wide scale. ${ }^{22-24}$ Thought also needs to be given to the membership of such a body. Time availability, clinical background, regional and rural representation, data experts, patient safety managers and key medical and nursing opinion leaders are all important ingredients for success.

Competing interests None.

Patient consent Obtained.

Provenance and peer review Not commissioned; externally peer reviewed.

\section{REFERENCES}

1. Leape $\mathbf{L}$, Berwick D. Five years after to Err is Human. What have we learned? JAMA 2005:293:2384-90.

2. Loeb JM. The current state of performance measurement in healthcare. Int J Oual Health Care 2004;6:15-19.

3. Berwick D. Error today and error tomorrow. N Engl J Med 2003;348:2570-2.

4. Wu A, Lipshutz K, Pronovost P. Effectiveness of root cause analysis in medicine. JAMA 2008:299:685-7.

5. Stanhope N, Crowley-Murphy M, Vincent C, et al. An evaluation of adverse incident reporting. J Eval Clin Pract 1999;5:5-12.

6. Woloshynowych M, Rogers S, Taylor-Adams S, et al. The investigation and analysis of critical incidents and adverse events in healthcare. Health Technol Assess 2005; 9:1-158.

7. Rogers S, Taylor-Adams S, Woloshynowych M. Techniques used in the investigation analysis of critical incidents in healthcare. In: Walshe K, Boaden R, eds. Patient safety: research into practice. Maidenhead: Open University Press, 2005:130-43.

8. Boyer M. Root cause analysis in perinatal care: health care professionals creating safer health care systems. J Perinat Neonatal Nurs 2001;15:40-54.

9. Bagian J, Gosbee J, Lee $\mathrm{C}$, et al. The veterans affairs root cause analysis system in action. Jt Comm J Qual Improv 2002;28:531-45.

10. Vincent C. Analysis of clinical incidents; a window on the system not a search for root causes. Qual Saf Health Care 2004:13:242-3.

11. Percarpio K, Watts $V$, Weeks W. The effectiveness of root cause analysis: what does the literature tell us? Jt Comm J Qual Patient Saf 2008;34:391-8.

12. Braithwaite J, Westbrook M, Mallock, et al. Experiences of health professionals who conducted root cause analyses after undergoing a safety improvement program. Qual Saf Health care 2006;15:393-9.

13. Lewis R, Fletcher M. Implementing a national strategy for patient safety: lessons from the National Health Service in England. Qual Saf Health Care 2005;14:135-9.

14. Wakefield J. Root cause analysis legislation-what is it and why is it needed? Old Nurse 2007;26:22

15. Vincent C, Reason J. Human factors approaches in medicine. In: Rosenthal M, Mulcahy L, Lloyd-Bostock S, eds. Medical mishaps; pieces of the puzzle. Buckingham: Open University Press, 1999:39-56.

16. Raboel I, Egbert J, Bjorn B. Root cause analysis in Copenhagen 2002-2008-an overview of the quantitative data. Poster presentation. Paris: International Quality Forum, 2008

17. Vincent C. Understanding and responding to adverse events. N Eng/ J Med 2003;11:1051-6.

18. Vincent C. Incident reporting and patient safety. BMJ 2007;334:51.

19. Hughes D. Root cause analysis: bridging the gap between ideas and execution. Washington DC, USA: National Centre for Patient Safety Topics in Patient Safety, 2006:1-2.

20. Kruk R. Patient safety and Clinical Quality program. First Report on incident management in the NSW public Health system 2003-05. North Sydney: NSW Department of health, 2005.

21. Garling P. Report of the Special Commission of Enquiry into Acute care Services in NSW, 2008.

22. Reason J. Beyond the organizational accident; the need for 'error wisdom' on the frontline. Qual Saf Health Care 2004;13:ii28-33.

23. Woodward S. Achieving a safer health service: Part 3. Investigating root causes and formulating solutions. Prof Nurse 2004;19:390-4.

24. Wallace M. From root cause analysis to safer systems. International comparisons of nationally sponsored healthcare staff training programmes. Qual Saf health Care 2006;15:388-91. 